\title{
On the origin of reentrance in 2D Josephson Junction Arrays
}

\author{
F.M. Araujo-Moreira, ${ }^{1}$ W. Maluf, ${ }^{1}$ and S. Sergeenkov ${ }^{1,2}$ \\ ${ }^{1}$ Departamento de Física e Engenharia \\ Física, Grupo de Materiais e Dispositivos, Centro \\ Multidisciplinar para o Desenvolvimento de Materiais Cerâmicos, \\ Universidade Federal de São Carlos, São Carlos, SP, 13565-905 Brazil \\ ${ }^{2}$ Bogoliubov Laboratory of Theoretical Physics, Joint Institute for Nuclear Research, \\ Dubna 141980, Moscow Region, Russia
}

(November 15, 2018)

A comparative study of the magnetic properties of shunted and unshunted two-dimensional Josephson junction arrays (2D-JJA) is presented. Using a singleplaquette approximation of the 2D-JJA model, we were able to successfully fit all our experimental data (for the temperature, AC and DC field dependencies of susceptibility) and demonstrate that the dynamic reentrance of AC susceptibility is directly linked to the value of the Stewart-McCumber parameter $\beta_{C}$. Based on extensive numerical simulations, a phase diagram $\beta_{C}-\beta_{L}$ is plotted which demarcates the border between the reentrant and non-reentrant behavior in the arrays.

\section{INTRODUCTION}

According to the current paradigm, paramagnetic Meissner effect (PME) [1-6] can be related to the presence of $\pi$-junctions [7], either resulting from the presence of magnetic impurities in the junction $[8,9]$ or from unconventional pairing symmetry [10]. Other possible explanations of this phenomenon are based on flux trapping [11] and flux compression effects [12] including also an important role of the surface of the sample [3]. Besides, in the experiments with unshunted 2D-JJA, we have previously reported [13] that PME manifests itself through a dynamic reentrance (DR) of the AC magnetic susceptibility as a function of temperature. These results have been further corroborated by Nielsen et al. [14] and De Leo et al. [15] who argued that PME can be simply related to magnetic screening in multiply connected superconductors. So, the main question is: which parameters are directly responsible for the presence (or absence) of DR in artificially prepared arrays?

Previously (also within the single plaquette approximation), Barbara et al. [13] have briefly discussed the effects of varying $\beta_{L}$ on the observed dynamic reentrance with the main emphasis on the behavior of 2D-JJA samples with high (and fixed) values of $\beta_{C}$. However, to our knowledge, up to date no systematic study (either experimental or theoretical) has been done on how the $\beta_{C}$ value itself affects the reentrance behavior. In the present work, by a comparative study of the magnetic properties of shunted and unshunted 2D-JJA, we propose an answer to this open question. Namely, by using experimental and theoretical results, we will demonstrate that only arrays with sufficiently large value of the StewartMcCumber parameter $\beta_{C}$ will exhibit the dynamic reentrance behavior (and hence PME).

\section{EXPERIMENTAL RESULTS}

To measure the complex AC susceptibility in our arrays we used a high-sensitive home-made susceptometer based on the so-called screening method in the reflection configuration [16-18]. The experimental system was calibrated by using a high-quality niobium thin film. Previously [18], we have shown that the calibrated output complex voltage of the experimental setup corresponds to the complex AC susceptibility.

To experimentally investigate the origin of the reentrance, we have measured $\chi^{\prime}(T)$ for three sets of shunted and unshunted samples obtained from different makers (Westinghouse and Hypress) under the same conditions of the amplitude of the excitation field $h_{a c}(1 m O e<$ $\left.h_{a c}<10 O e\right)$, external magnetic field $H_{d c}\left(0<H_{d c}<\right.$ $5000 e)$ parallel to the plane of the sample, and frequency of $\mathrm{AC}$ field $\omega=2 \pi f$ (fixed at $f=20 \mathrm{kHz}$ ). Unshunted 2D-JJAs are formed by loops of niobium islands linked through $\mathrm{Nb}-\mathrm{AlO} \mathrm{O}_{x}-\mathrm{Nb}$ Josephson junctions while shunted 2D-JJAs have a molybdenum shunt resistor (with $R_{s h} \simeq 2.2 \Omega$ ) short-circuiting each junction (see Fig.1). Both shunted and unshunted samples have rectangular geometry and consist of $100 \times 150$ tunnel junctions. The unit cell for both types of arrays has square geometry with lattice spacing $a \simeq 46 \mu \mathrm{m}$ and a single junction area of $5 \times 5 \mu m^{2}$. The critical current density for the junctions forming the arrays is about $600 \mathrm{~A} / \mathrm{cm}^{2}$ at $4.2 \mathrm{~K}$. Besides, for the unshunted samples $\beta_{C}(4.2 K) \simeq 30$ and $\beta_{L}(4.2 K) \simeq 30$, while for shunted samples $\beta_{C}(4.2 K) \simeq 1$ and $\beta_{L}(4.2 K) \simeq 30$ where $[19] \beta_{L}(T)=\frac{2 \pi L I_{C}(T)}{\Phi_{0}}$ and $\beta_{C}(T)=\frac{2 \pi C_{J} R_{J}^{2} I_{C}(T)}{\Phi_{0}}$. Here, $C_{J} \simeq 0.58 p F$ is the capacitance, $R_{J} \simeq 10.4 \Omega$ the quasi-particle resistance (of unshunted array), and $I_{C}(4.2 K) \simeq 150 \mu \mathrm{A}$ critical current of the Josephson junction. $\Phi_{0}$ is the quantum of 
magnetic flux. The parameter $\beta_{L}$ is proportional to the number of flux quanta that can be screened by the maximum critical current in the junctions, while the StewartMcCumber parameter $\beta_{C}$ basically reflects the quality of the junctions in arrays.

It is well established that both magnetic and transport properties of any superconducting material can be described via a two-component response [20], the intragranular (associated with the grains exhibiting bulk superconducting properties) and intergranular (associated with weak-link structure) contributions [21,22]. Likewise, artificially prepared JJAs (consisting of superconducting islands, arranged in a symmetrical periodic lattice and coupled by Josephson junctions) will produce a similar response [23].

Since our shunted and unshunted samples have the same value of $\beta_{L}$ and different values of $\beta_{C}$, it is possible to verify the dependence of the reentrance effect on the value of the Stewart-McCumber parameter. For the unshunted 2D-JJA (Fig. 2a) we have found that for an $\mathrm{AC}$ field lower than $50 \mathrm{mOe}$ (when the array is in the Meissner-like state) the behavior of $\chi^{\prime}(T)$ is quite similar to homogeneous superconducting samples, while for $h_{a c}>50 m O e$ (when the array is in the mixedlike state with practically homogeneous flux distribution) these samples exhibit a clear reentrant behavior of susceptibility [13]. At the same time, the identical experiments performed on the shunted samples produced no evidence of any reentrance for all values of $h_{a c}$ (see Fig. $2 \mathrm{~b}$ ). It is important to point out that the analysis of the experimentally obtained imaginary component of susceptibility $\chi^{\prime \prime}(T)$ shows that for the highest AC magnetic field amplitudes (of about 200mOe) dissipation remains small. Namely, for typical values of the AC amplitude, $h_{a c}=100 \mathrm{mOe}$ (which corresponds to about 10 vortices per unit cell) the imaginary component is about 15 times smaller than its real counterpart. Hence contribution from the dissipation of vortices to the observed phenomena can be safely neglected.

To further study this unexpected behavior we have also performed experiments where we measure $\chi^{\prime}(T)$ for different values of $H_{d c}$ keeping the value of $h_{a c}$ constant. The influence of DC fields on reentrance in unshunted samples is shown in Fig. 3. On the other hand, the shunted samples still show no signs of reentrance, following a familiar pattern of field-induced gradual diminishing of superconducting phase (very similar to a zero DC field flat-like behavior seen in Fig.2b).

To understand the influence of DC field on reentrance observed in unshunted arrays, it is important to emphasize that for our sample geometry this parallel field suppresses the critical current $I_{C}$ of each junction without introducing any detectable flux into the plaquettes of the array. Thus, a parallel DC magnetic field allows us to vary $I_{C}$ independently from temperature and/or applied perpendicular AC field. The measurements show (see Fig.3) that the position of the reentrance is tuned by $H_{d c}$. We also observe that the value of temperature $T_{\min }$ (at which $\chi^{\prime}(T)$ has a minimum) first shifts towards lower temperatures as we raise $H_{d c}$ (for small DC fields) and then bounces back (for higher values of $\left.H_{d c}\right)$. This non-monotonic behavior is consistent with the weakening of $I_{C}(T)$ and corresponds to Fraunhoferlike dependence of the Josephson junction critical current on DC magnetic field applied in the plane of the junction. We measured $I_{C}$ from transport current-voltage characteristics, at different values of $H_{d c}$ at $T=4.2 \mathrm{~K}$ and found that $\chi^{\prime}(T=4.2 K)$, obtained from the isotherm $T=4.2 K$ (similar to that given in Fig.3), shows the same Fraunhofer-like dependence on $H_{d c}$ as the critical current $I_{C}\left(H_{d c}\right)$ of the junctions forming the array (see Fig.4). This gives further proof that only the junction critical current is varied in this experiment. This also indicates that the screening currents at low temperature (i.e., in the reentrant region) are proportional to the critical currents of the junctions. In addition, this shows an alternative way to obtain $I_{C}\left(H_{d c}\right)$ dependence in big arrays. And finally, a sharp Fraunhofer-like pattern observed in both arrays clearly reflects a rather strong coherence (with negligible distribution of critical currents and sizes of the individual junctions) which is based on highly correlated response of all single junctions forming the arrays, thus proving their high quality. Such a unique behavior of Josephson junctions in our samples provides a necessary justification for suggested theoretical interpretation of the obtained experimental results. Namely, based on the above-mentioned properties of our arrays, we have found that practically all the experimental results can be explained by analyzing the dynamics of just a single unit cell in the array.

\section{THEORETICAL INTERPRETATION AND NUMERICAL SIMULATIONS}

To understand the different behavior of the AC susceptibility observed in shunted and unshunted 2D-JJAs, in principle one would need to analyze in detail the flux dynamics in these arrays. However, as we have previously reported [13], because of the well-defined periodic structure of our arrays (with no visible distribution of junction sizes and critical currents), it is reasonable to expect that the experimental results obtained from the magnetic properties of our 2D-JJAs can be quite satisfactory explained by analyzing the dynamics of a single unit cell (plaquette) of the array. An excellent agreement between a single-loop approximation and the observed behavior (seen through the data fits) justifies a posteriori our assumption. It is important to mention that the idea to use a single unit cell to qualitatively understand PME was first suggested by Auletta et al. [24]. They simulated the field-cooled DC magnetic susceptibility of 
a single-junction loop and found a paramagnetic signal at low values of external magnetic field.

In our calculations and numerical simulations, the unit cell is a loop containing four identical Josephson junctions and the measurements correspond to the zero-field cooling (ZFC) AC magnetic susceptibility. We consider the junctions of the single unit cell as having capacitance $C_{J}$, quasi-particle resistance $R_{J}$ and critical current $I_{C}$. We have used this simple four-junctions model to study the magnetic behavior of our 2D-JJA by calculating the AC complex magnetic susceptibility $\chi=\chi^{\prime}+i \chi^{\prime \prime}$ as a function of $T, \beta_{C}$ and $\beta_{L}$. Specifically, shunted samples are identified through low values of the McCumber parameter $\left(\beta_{C} \approx 1\right)$ while high values $\left(\beta_{C} \gg 1\right)$ indicate an unshunted 2D-JJA.

If we apply an AC external field $B_{a c}(t)=\mu_{0} h_{a c} \cos \omega t$ normally to the 2D-JJA and a DC field $B_{d c}=\mu_{0} H_{d c}$ parallel to the array, then the total magnetic flux $\Phi(t)$ threading the four-junction superconducting loop is given by $\Phi(t)=\Phi_{e x t}(t)+L I(t)$ where $L$ is the loop inductance, $\Phi_{e x t}(t)=S B_{a c}(t)+l d B_{d c}$ is the flux related to the applied magnetic field (with $l \times d$ being the size of the single junction area, and $S \simeq a^{2}$ being the projected area of the loop), and the circulating current in the loop reads

$$
I(t)=I_{C}(T) \sin \phi_{i}(t)+\frac{\Phi_{0}}{2 \pi R_{J}} \frac{d \phi_{i}}{d t}+\frac{C_{J} \Phi_{0}}{2 \pi} \frac{d^{2} \phi_{i}}{d t^{2}}
$$

Here $\phi_{i}(t)$ is the gauge-invariant superconducting phase difference across the $i$ th junction, and $\Phi_{0}$ is the magnetic flux quantum.

Since the inductance of each loop is $L=\mu_{0} a \simeq 64 p H$ and the critical current of each junction is $I_{C} \simeq 150 \mu \mathrm{A}$, for the mixed-state region (above $50 \mathrm{mOe}$ ) we can safely neglect the self-field effects because in this region $L I(t)$ is always smaller than $\Phi_{\text {ext }}(t)$. Besides, since the length $l$ and the width $w$ of each junction in our array is smaller than the Josephson penetration depth $\lambda_{J}=$ $\sqrt{\Phi_{0} / 2 \pi \mu_{0} d j_{c 0}}$ (where $j_{c 0}$ is the critical current density of the junction, and $d=2 \lambda_{L}+\xi$ is the size of the contact area with $\lambda_{L}(T)$ being the London penetration depth of the junction and $\xi$ an insulator thickness), namely $l \simeq w \simeq 5 \mu \mathrm{m}$ and $\lambda_{J} \simeq 20 \mu \mathrm{m}$ (using $j_{c 0} \simeq 600 \mathrm{~A} / \mathrm{cm}^{2}$ and $\lambda_{L} \simeq 39 \mathrm{~nm}$ for $N b$ at $T=4.2 \mathrm{~K}$ ), we can adopt the small-junction approximation [19] for the gauge-invariant superconducting phase difference across the $i$ th junction (for simplicity we assume as usual [13] that $\phi_{1}=\phi_{2}=$ $\left.\phi_{3}=\phi_{4} \equiv \phi_{i}\right)$

$$
\phi_{i}(t)=\phi_{0}\left(H_{d c}\right)+\frac{2 \pi B_{a c}(t) S}{\Phi_{0}}
$$

where $\phi_{0}\left(H_{d c}\right)=\phi_{0}(0)+2 \pi \mu_{0} H_{d c} d l / \Phi_{0}$ with $\phi_{0}(0)$ being the initial phase difference.

To properly treat the magnetic properties of the system, let us introduce the following Hamiltonian

$$
\mathcal{H}(t)=J \sum_{i=1}^{4}\left[1-\cos \phi_{i}(t)\right]+\frac{1}{2} L I^{2}(t)
$$

which describes the tunneling (first term) and inductive (second term) contributions to the total energy of a single plaquette. Here, $J(T)=\left(\Phi_{0} / 2 \pi\right) I_{C}(T)$ is the Josephson coupling energy.

The real part of the complex AC susceptibility is defined as

$$
\chi^{\prime}\left(T, h_{a c}, H_{d c}\right)=\frac{\partial M}{\partial h_{a c}}
$$

where

$$
M\left(T, h_{a c}, H_{d c}\right)=-\frac{1}{V}\left\langle\frac{\partial \mathcal{H}}{\partial h_{a c}}\right\rangle
$$

is the net magnetization of the plaquette. Here $V$ is the sample's volume, and $\langle\ldots\rangle$ denotes the time averaging over the period $2 \pi / \omega$, namely

$$
<A>=\frac{1}{2 \pi} \int_{0}^{2 \pi} d(\omega t) A(t)
$$

Taking into account the well-known [25] analytical approximation of the BCS gap parameter (valid for all temperatures), $\Delta(T)=\Delta(0) \tanh \left(2.2 \sqrt{\frac{T_{c}-T}{T}}\right)$ for the explicit temperature dependence of the Josephson critical current

$$
I_{C}(T)=I_{C}(0)\left[\frac{\Delta(T)}{\Delta(0)}\right] \tanh \left[\frac{\Delta(T)}{2 k_{B} T}\right]
$$

we successfully fitted all our data using the following set of parameters: $\phi_{0}(0)=\frac{\pi}{2}$ (which corresponds to the maximum Josephson current within a plaquette), $\beta_{L}(0)=32$, $\beta_{C}(0)=32$ (for unshunted array) and $\beta_{C}(0)=1.2$ (for shunted array). The corresponding fits are shown by solid lines in Figs. 2 and 3 for the experimental values of $\mathrm{AC}$ and DC field amplitudes.

In the mixed-state region and for low enough frequencies (this assumption is well-satisfied because in our case $\omega \ll \omega_{L R}$ and $\omega \ll \omega_{L C}$ where $\omega_{L R}=R / L$ and $\omega_{L C}=1 / \sqrt{L C}$ are the two characteristic frequencies of the problem) from Eqs.(3)-(6) we obtain the following approximate analytical expression for the susceptibility of the plaquette

$$
\begin{aligned}
\chi^{\prime}\left(T, h_{a c}, H_{d c}\right) & \simeq-\chi_{0}(T)\left[\beta_{L}(T) f_{1}(b) \cos \left(\frac{2 H_{d c}}{H_{0}}\right)\right. \\
& \left.+f_{2}(b) \sin \left(\frac{H_{d c}}{H_{0}}\right)-\beta_{C}^{-1}(T)\right]
\end{aligned}
$$

where $\chi_{0}(T)=\pi S^{2} I_{C}(T) / V \Phi_{0}, H_{0}=\Phi_{0} /\left(2 \pi \mu_{0} d l\right) \simeq$ $10 O e, f_{1}(b)=J_{0}(2 b)-J_{2}(2 b)$, and $f_{2}(b)=J_{0}(b)-$ $b J_{1}(b)-3 J_{2}(b)+b J_{3}(b)$ with $b=2 \pi S \mu_{0} h_{a c} / \Phi_{0}$ and $J_{n}(x)$ being the Bessel function of the $n$th order. 
Notice also that the analysis of Eq.(8) reproduces the observed Fraunhofer-like behavior of the susceptibility in applied DC field (see Fig.4) and the above-mentioned fine tuning of the reentrance effect (see also Ref.13). Indeed, according to Eq.(8) (and in agreement with the observations), for small $\mathrm{DC}$ fields the minimum temperature $T_{\min }$ (indicating the beginning of the reentrant transition) varies with $H_{d c}$ as follows, $1-T_{\min } / T_{C} \simeq H_{d c} / H_{0}$.

To further test our interpretation and verify the influence of the parameter $\beta_{C}$ on the reentrance, we have also performed extensive numerical simulations of the fourjunction model previously described but without a simplifying assumption about the explicit form of the phase difference based on Eq.(2). More precisely, we obtained the temperature behavior of the susceptibility by solving the set of equations responsible for the flux dynamics within a single plaquette and based on Eq.(1) for the total current $I(t)$, the equation for the total flux $\Phi(t)=\Phi_{\text {ext }}(t)+L I(t)$ and the flux quantization condition for four junctions, namely $\phi_{i}(t)=\frac{\pi}{2}\left(n+\frac{\Phi}{\Phi_{0}}\right)$ where $n$ is an integer. Both Euler and fourth-order RungeKutta integration methods provided the same numerical results. In Fig.5 we show the real component of the simulated susceptibility $\chi(T)$ corresponding to the fixed value of $\beta_{C}(T=4.2 K)=1$ (shunted samples) and different values of $\beta_{L}(T=4.2 K)=1,10,15,20,30,40,50,60,90$, 150 and 200 . As expected, for this low value of $\beta_{C}$ reentrance is not observed for any values of $\beta_{L}$. On the other hand, Fig.6 shows the real component of the simulated $\chi(T)$ but now using fixed value of $\beta_{L}(T=4.2 K)=30$ and different values of $\beta_{C}(T=4.2 K)=1,2,5,10,20$, 30 and 100 . This figure clearly shows that reentrance appears for values of $\beta_{C}>20$. In both cases we used $h_{a c}=70 m O e$. We have also simulated the curve for shunted $\left(\beta_{L}=30, \beta_{C}=1\right)$ and unshunted $\left(\beta_{L}=30\right.$, $\left.\beta_{C}=30\right)$ samples for different values of $h_{a c}$ (see Fig.7). In this case the values of the parameters $\beta_{L}$ and $\beta_{C}$ were chosen from our real 2D-JJA samples. Again, our simulations confirm that dynamic reentrance does not occur for low values of $\beta_{C}$, independently of the values of $\beta_{L}$ and $h_{a c}$.

The following comment is in order regarding some irregularities visibly seen in Figs.(5)-(7) around the transition regions from non-reentrant to reentrant behavior. It is important to emphasize that the above irregularities are just artifacts of the numerical simulations due to the conventional slow-converging real-time reiteration procedures [13]. They neither correspond to any experimentally observed behavior (within the accuracy of the measurements technique and data acquisition), nor they reflect any irregular features of the considered here theoretical model (which predicts a smooth temperature dependence seen through the data fits). As usual, to avoid this kind of artificial (non-physical) discontinuity, more powerful computers are needed.
Based on the above extensive numerical simulations, a resulting phase diagram $\beta_{C}-\beta_{L}$ (taken for $T=1 K$, $h_{a c}=70 \mathrm{mOe}$, and $\left.H_{d c}=0\right)$ is depicted in Fig.8 which clearly demarcates the border between the reentrant (white area) and non-reentrant (shaded area) behavior in the arrays for different values of $\beta_{L}(T)$ and $\beta_{C}(T)$ parameters at given temperature. In other words, if $\beta_{L}$ and $\beta_{C}$ parameters of any realistic array have the values inside the white area, this array will exhibit a reentrant behavior. In addition, this diagram shows that one can prepare a reentrance exhibiting array by changing one of the parameters (usually, it is much easier to change $\beta_{C}$ by tuning the shunt resistance rather than the geometry related inductance parameter $\beta_{L}$ ).

It is instructive to mention that a hyperbolic-like character of $\beta_{L}$ vs $\beta_{C}$ law (seen in Fig.8) is virtually present in the approximate analytical expression for the susceptibility of the plaquette given by Eq.(8) (notice however that this expression can not be used to produce any quantitative prediction because the neglected in Eq.(8) frequencyrelated terms depend on $\beta_{L}$ and $\beta_{C}$ parameters as well). A qualitative behavior of the envelope of the phase diagram (depicted in Fig.8) with DC magnetic field $H_{d c}$ (for $T=1 \mathrm{~K}$ and $h_{a c}=70 \mathrm{mOe}$ ), obtained using Eq.(8), is shown in Fig.9.

And finally, to understand how small values of $\beta_{C}$ parameter affect the flux dynamics in shunted arrays, we have analyzed the $\Phi_{t o t}\left(\Phi_{\text {ext }}\right)$ diagram. Similarly to those results previously obtained from unshunted samples [13], for a shunted sample at fixed temperature this curve is also very hysteretic (see Fig.10). In both cases, $\Phi_{\text {tot }}$ vs. $\Phi_{\text {ext }}$ shows multiple branches intersecting the line $\Phi_{t o t}=0$ which corresponds to diamagnetic states. For all the other branches, the intersection with the line $\Phi_{t o t}=\Phi_{e x t}$ corresponds to the boundary between diamagnetic states (negative values of $\chi^{\prime}$ ) and paramagnetic states (positive values of $\chi^{\prime}$ ). As we have reported before [13], for unshunted 2D-JJA at temperatures below $7.6 K$ the appearance of the first and third branches adds a paramagnetic contribution to the average value of $\chi^{\prime}$. When $\beta_{C}$ is small (shunted arrays), the analysis of these curves shows that there is no reentrance at low temperatures because in this case the second branch appears to be energetically stable, giving an extra diamagnetic contribution which overwhelms the paramagnetic contribution from subsequent branches. In other words, for low enough values of $\beta_{C}$ (when the samples are ZFC and then measured at small values of the magnetic field), most of the loops will be in the diamagnetic states, and no paramagnetic response is registered. As a result, the flux quanta cannot get trapped into the loops even by the following field-cooling process in small values of the magnetic field. In this case the superconducting phases and the junctions will have the same diamagnetic response and the resulting measured value of the magnetic susceptibility will be negative (i.e., diamagnetic) as well. On 
the other hand, when $\beta_{C}$ is large enough (unshunted arrays), the second branch becomes energetically unstable, and the average response of the sample at low temperatures is paramagnetic (Cf. Fig.7 from Ref. [13]).

In conclusion, our experimental and theoretical results have demonstrated that the reentrance phenomenon (and concomitant PME) in artificially prepared Josephson Junction Arrays is related to the damping effects associated with the Stewart-McCumber parameter $\beta_{C}$. Namely, reentrant behavior of AC susceptibility takes place in the underdamped (unshunted) array (with large enough value of $\beta_{C}$ ) and totally disappears in overdamped (shunted) arrays.

\section{ACKNOWLEDGMENTS}

We thank P. Barbara, C.J. Lobb, R.S. Newrock, and A. Sanchez for useful discussions. S.S. and F.M.A.M. gratefully acknowledge financial support from Brazilian Agency FAPESP under grant 03/00296-5.

[1] W. Braunisch, N. Knauf, S. Neuhausen, A. Grutz, A. Koch, B. Roden, D. Khomskii, D. Wohlleben, Phys. Rev. Lett. 68, 1908 (1992)

[2] M.S. Li, Phys. Rep. 376, 133 (2003)

[3] P. Kostic, B. Veal, A.P. Paulikas, U. Welp, V.R. Todt, C. Gu, U. Geiser, J.M. Williams, K.D. Carlson, R.A. Klemm, Phys. Rev. B 53, 791 (1996)

[4] W.A. Ortiz, P.N. Lisboa-Filho, W.A.C. Passos, F.M. Araujo-Moreira, Physica C 361, 267 (2001)

[5] W.A.C. Passos, P.N. Lisboa-Filho, W.A. Ortiz, Physica C 341-348, 2723 (2000)

[6] R. Lucht, H.v. Löhneysen, H. Claus, M. Kläser, G. Müller-Vogt, Phys. Rev. B 52, 9724 (1995)

[7] M.S. Li, D. Dominguez, Phys. Rev. B 62, 14554 (2000)

[8] L.N. Bulaevskii, V.V. Kuzii, A.A. Sobyanin, JETP Lett. 25, 290 (1977)

[9] F.V. Kusmartsev, Phys. Rev. Lett. 69, 2268 (1992)

[10] H. Kawamura, M.S. Li, Phys. Rev. B 54, 619 (1996)

[11] D.X. Chen, A. Sanchez, A. Hernando, Physica C 250, 107 (1995)

[12] A. Terentiev, D.B. Watkins, L.E. De Long, D.J. Morgan, J.B. Ketterson, Phys. Rev. B 60, R761 (1999)

[13] F.M. Araujo-Moreira, P. Barbara, A.B. Cawthorne, C.J. Lobb, Phys. Rev. Lett. 78, 4625 (1997); P. Barbara, F.M. Araujo-Moreira, A.B. Cawthorne, C.J. Lobb, Phys. Rev. B 60, 7489 (1999)

[14] A.P. Nielsen, A.B. Cawthorne, P. Barbara, F.C. Wellstood, C.J. Lobb, Phys. Rev. B 62, 14380 (2000)

[15] C. De Leo, G. Rotoli, P. Barbara, A.P. Nielsen, C.J. Lobb, Phys. Rev. B 64, 14518 (2001); C. De Leo, G. Rotoli, Phys. Rev. Lett. 89, 167001 (2002)
[16] Magnetic Susceptibility of Superconductors and Other Spin Systems, edited by R.A. Hein, T.L. Francavilla, and D.H. Liebenberg, (Plenum Press, New York, 1992)

[17] J.L. Jeanneret, G.A. Gavilano, A. Racine, Ch. Leemann, P. Martinoli, Appl. Phys. Lett. 55, 2336 (1989)

[18] F.M. Araujo-Moreira, P. Barbara, A.B. Cawthorne, C.J. Lobb, in Studies of High temperature Superconductors, edited by A.V. Narlikar (Nova Science Publishers, New York, 2002), vol. 43, pp. 227-254

[19] A. Barone, G. Paterno, Physics and Applications of the Josephson Effect (A Wiley-Interscience Publisher, New York, 1982)

[20] F.M. Araujo-Moreira, O.F. de Lima, W.A. Ortiz, Physica C 240-245, 3205 (1994); F.M. Araujo-Moreira O.F. de Lima, W.A. Ortiz, J. Appl. Phys. 80, 6, 3390 (1996); F.M. Araujo-Moreira O.F. de Lima, W.A. Ortiz, Physica C 311, 98 (1999)

[21] W.A.C. Passos, P.N. Lisboa-Filho, R. Caparroz, C.C. de Faria, P.C. Venturini, F.M. Araujo-Moreira, S. Sergeenkov, W.A. Ortiz, Physica C 354, 189 (2001)

[22] R.S. Newrock, C.J. Lobb, U. Geigenmuller, M. Octavio, Solid State Physics 54, 263 (2000)

[23] P. Martinoli, C. Leeman, J. Low Temp. Phys. 118, 699 (2000)

[24] C. Auletta, P. Caputo, G. Costabile, R. de Luca, S. Pase, A. Saggese, Physica C 235-240, 3315 (1994); C. Auletta, G. Raiconi, R. de Luca, S. Pase, Phys. Rev. B 51, 12844 (1nnm
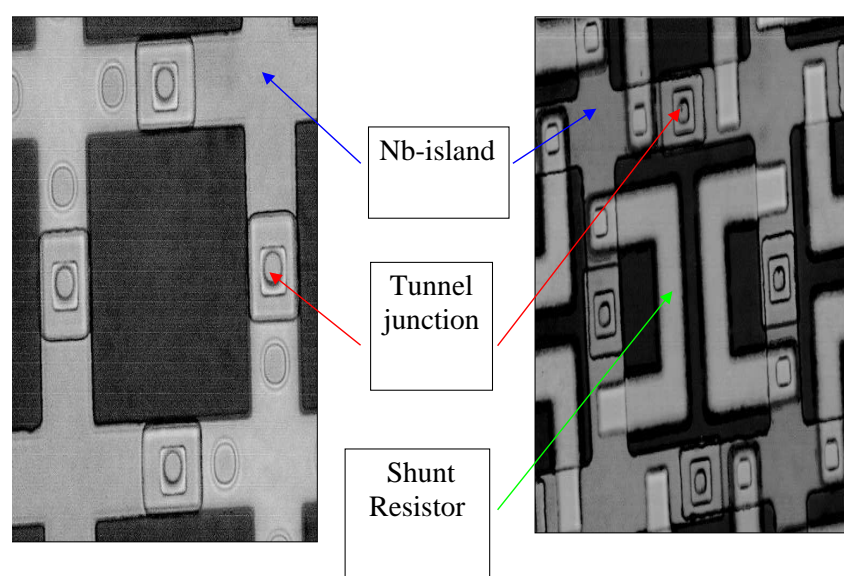

FIG. 1. Left: photograph of the unshunted array; right: photograph of the shunted array. 

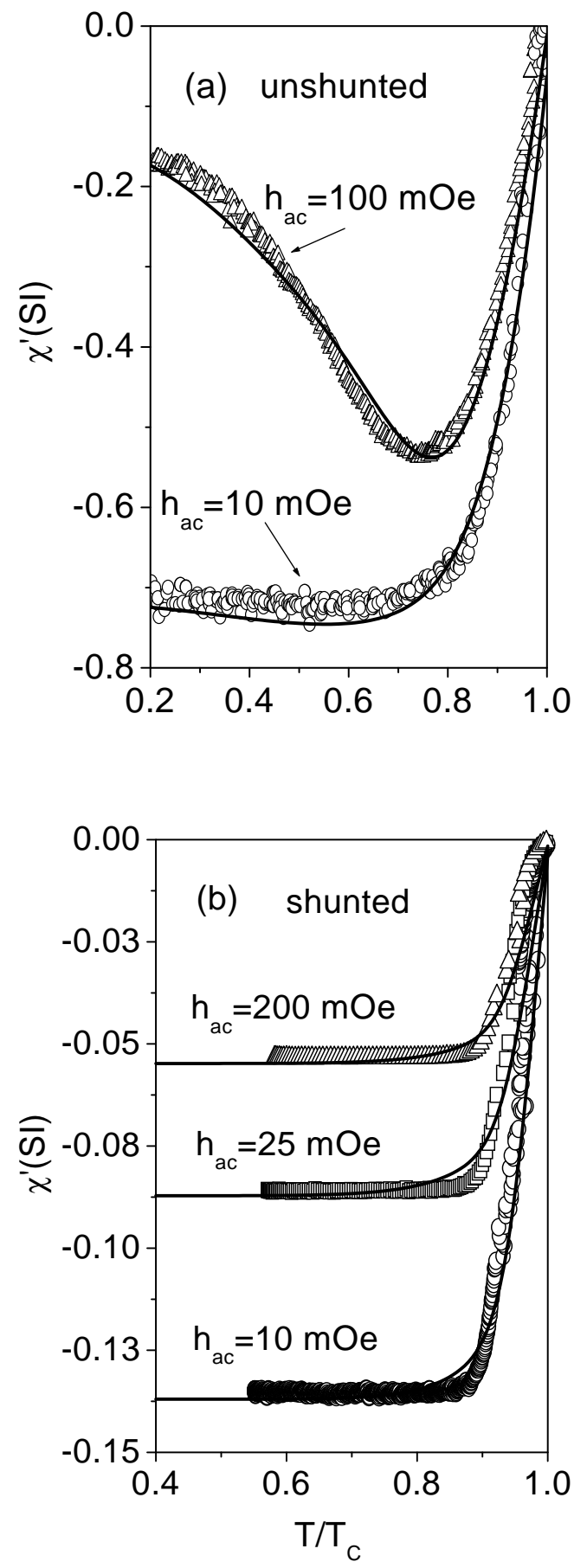

FIG. 2. Experimental results for $\chi^{\prime}\left(T, h_{a c}, H_{d c}\right)$ : (a) unshunted 2D-JJA for $h_{a c}=10$ and 100mOe; (b) shunted 2D-JJA for $h_{a c}=10,25$, and $200 m O e$. In all these experiments $H_{d c}=0$. Solid lines are the best fits (see text).
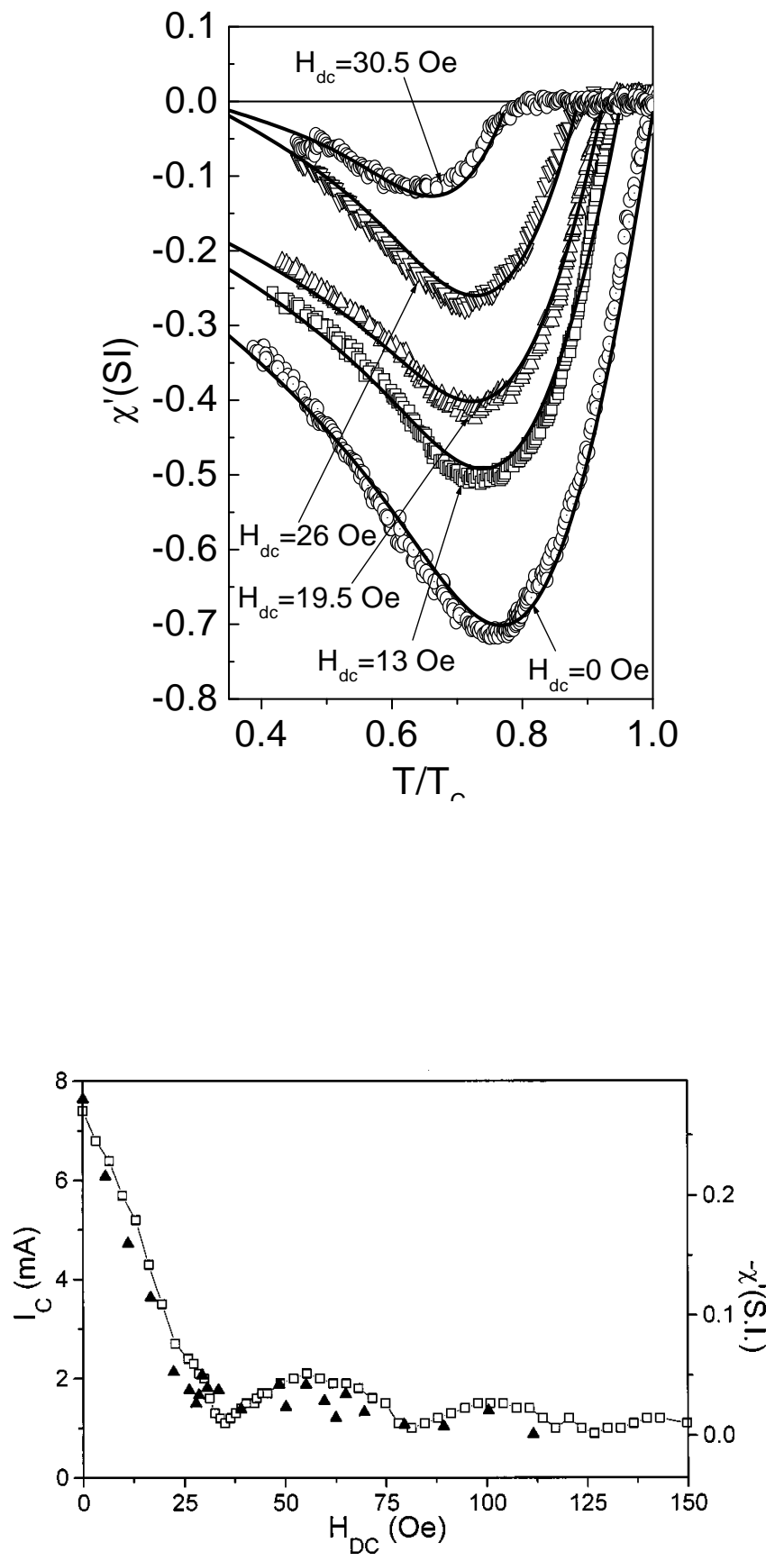

FIG. 4. The critical current $I_{C}$ (open squares) and the real part of AC susceptibility $\chi^{\prime}$ (solid triangles) as a function of DC field $H_{d c}$ for $T=4.2 K$ (from Ref.13). 


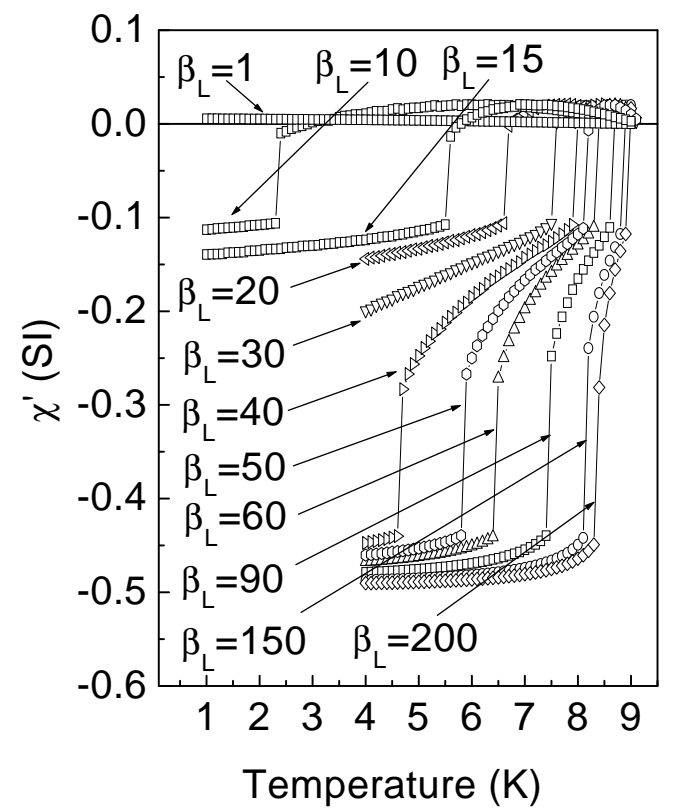

FIG. 5. Numerical simulation results for $h_{a c}=70 \mathrm{mOe}$, $H_{d c}=0, \beta_{C}(T=4.2 K)=1$ and for different values of $\beta_{L}(T=4.2 K)$ based on Eqs.(4)-(7).

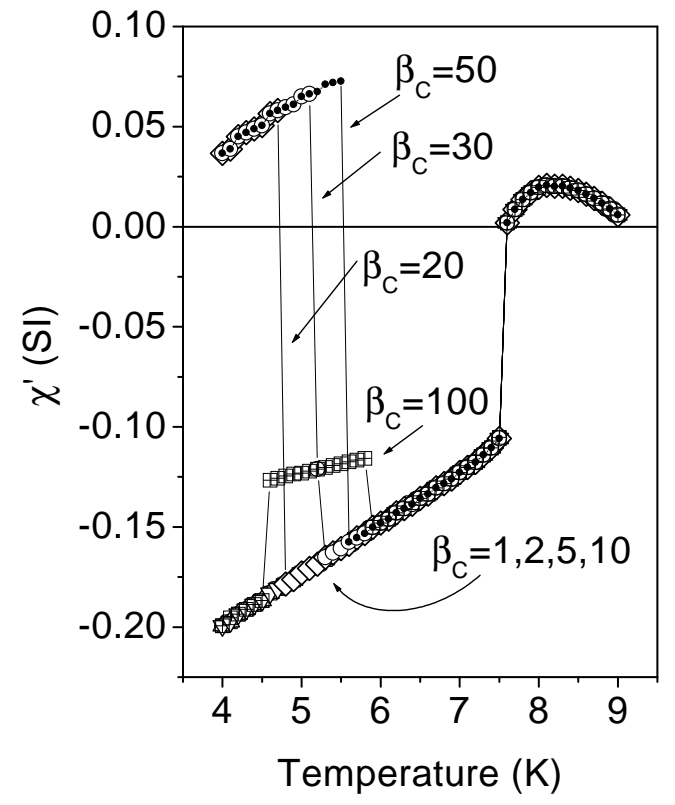

FIG. 6. Numerical simulation results for $h_{a c}=70 \mathrm{mOe}$, $H_{d c}=0, \beta_{L}(T=4.2 K)=30$ and for different values of $\beta_{C}(T=4.2 K)$ based on Eqs.(4)-(7).
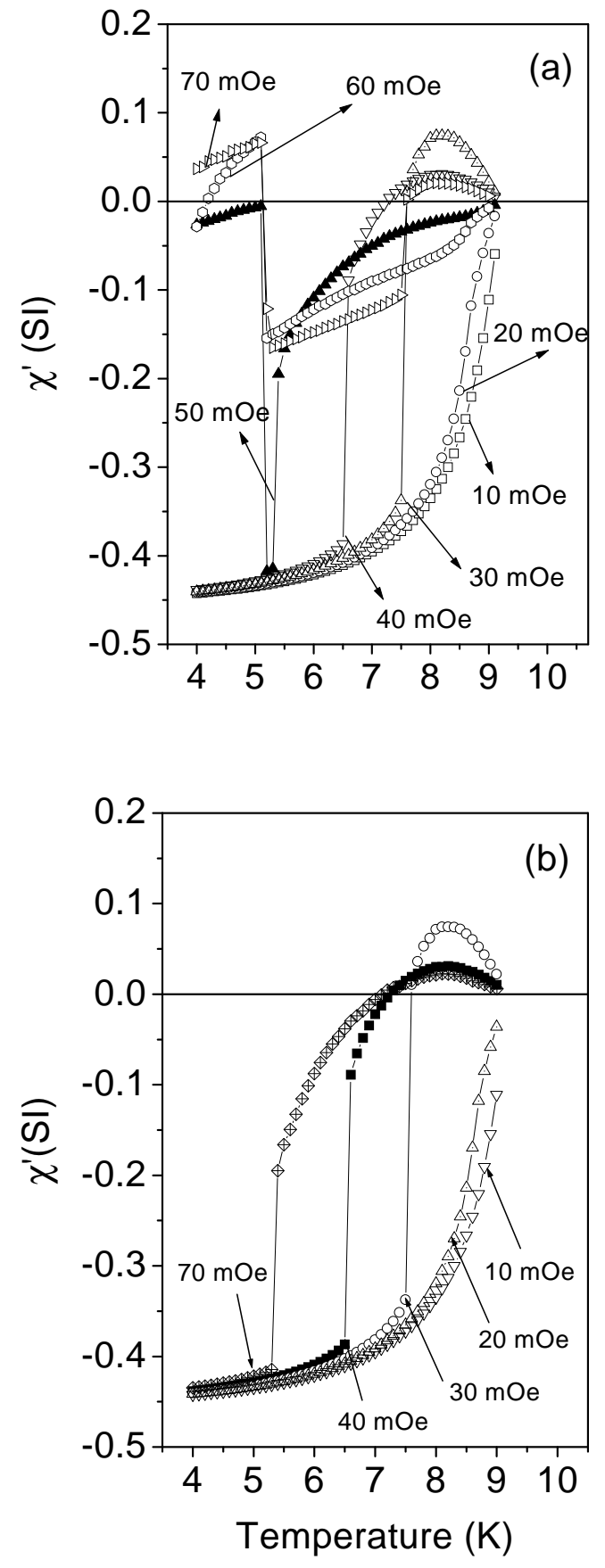

FIG. 7. Curves of the simulated susceptibility (for $H_{d c}=0$ and for different values of $h_{a c}$ ) corresponding to (a) unshunted 2D-JJA with $\beta_{L}(T=4.2 K)=30$ and $\beta_{C}(T=4.2 K)=30$; (b) shunted 2D-JJA with $\beta_{L}(T=4.2 K)=30$ and $\beta_{C}(T=4.2 K)=1$. 


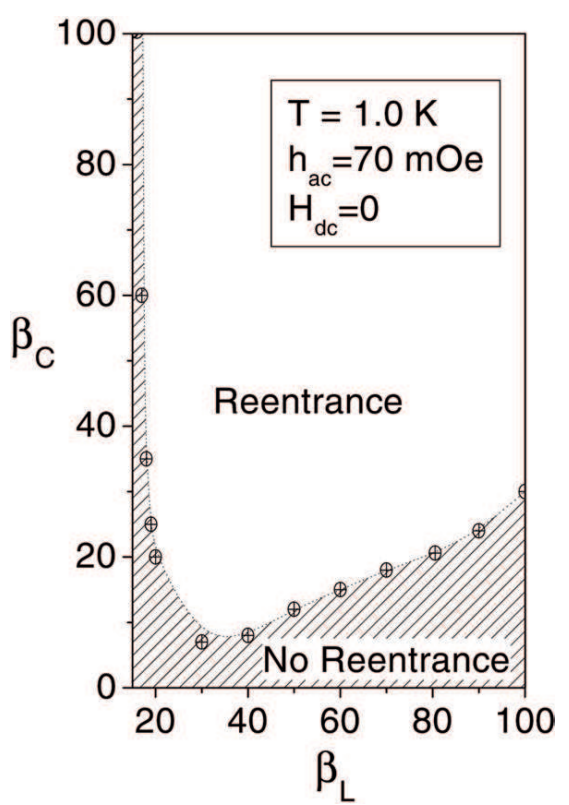

FIG. 8. Numerically obtained phase diagram (taken for $T=1 K, h_{a c}=70 m O e$, and $\left.H_{d c}=0\right)$ which shows the border between the reentrant (white area) and non-reentrant (shaded area) behavior in the arrays for different values of $\beta_{L}$ and $\beta_{C}$ narameters

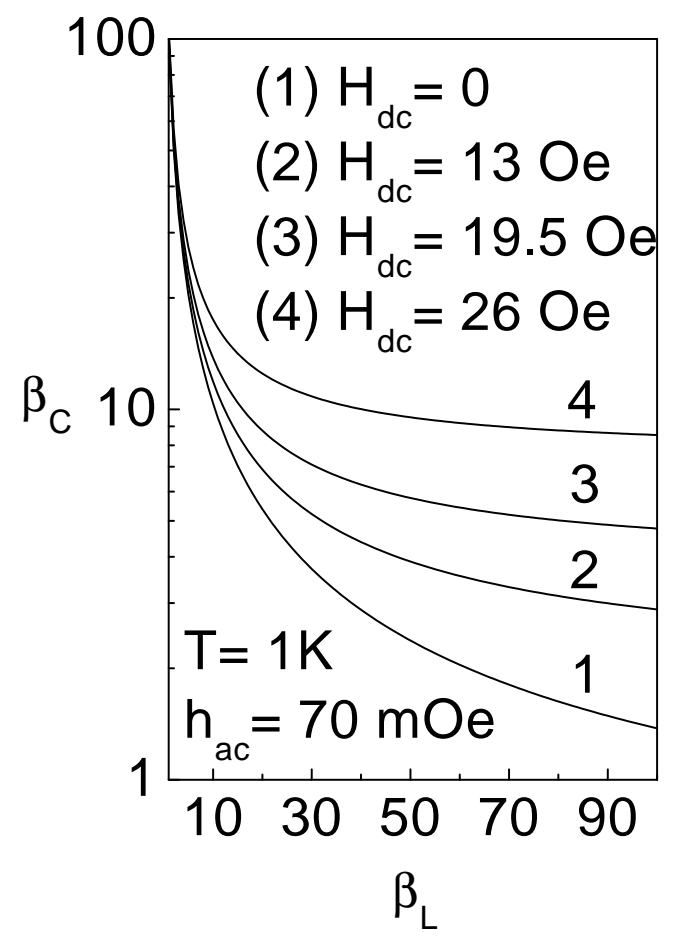

FIG. 9. A qualitative behavior of the envelope of the phase diagram (shown in previous figure) with DC magnetic field $H_{d c}$ (for $T=1 \mathrm{~K}$ and $h_{a c}=70 \mathrm{mOe}$ ) obtained from Eq.(8).

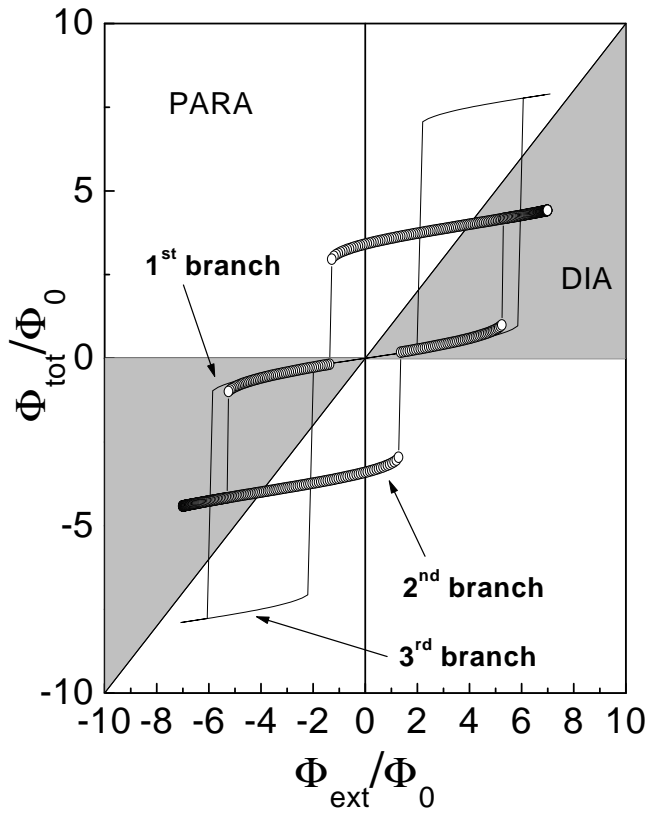

FIG. 10. Numerical simulation results showing $\Phi_{\text {tot }}$ vs. $\Phi_{\text {ext }}$ for shunted 2D-JJA with $\beta_{L}(T=4.2 K)=30$ and $\beta_{C}(T=4.2 K)=1$. 